\title{
Procedure to characterize microroughness of optical thin films: application to ion-beam-sputtered vacuum-ultraviolet coatings
}

\author{
Josep Ferré-Borrull, Angela Duparré, and Etienne Quesnel
}

\begin{abstract}
A method for characterizing the microroughness of samples in optical coating technology is developed. Measurements over different spatial-frequency ranges are composed into a single power spectral density (PSD) covering a large bandwidth. This is followed by the extraction of characteristic parameters through fitting of the PSD to a suitable combination of theoretical models. The method allows us to combine microroughness measurements performed with different techniques, and the fitting procedure can be adapted to any behavior of a combined PSD. The method has been applied to a set of ion-beamsputtered fluoride vacuum-UV coatings with increasing number of alternative low- and high-index layers. Conclusions about roughness development and microstructural growth are drawn. (C) 2001 Optical Society of America

OCIS codes: $310.6870,180.5810,120.6660$.
\end{abstract}

\section{Introduction}

With the ongoing trend of optical lithography toward ever-decreasing wavelengths, the interest in the study of the microroughness of optical coating components has taken a new perspective because of its crucial influence on the scattering losses of deep-UV and vacuum-UV (VUV) coatings. ${ }^{1,2}$ This makes residual roughnesses a dominant scatter source and a technological problem that has to be engineered if high-quality components are to be achieved.

Within this framework, new concepts for the optimization of coatings that take into account specific problems relevant only at these shorter wavelengths have to be developed. Among these concepts, the development of accurate modeling of microroughness of optical surfaces is of particular interest. Power spectral density (PSD) ${ }^{3,4}$ functions offer a comprehensive description of the surface statistics and are a

J. Ferré-Borrull and A. Duparré (duparre@iof.fhg.de) are with the Fraunhofer Institute for Applied Optics and Precision Engineering, Schillerstrasse 1, Jena D-07745, Germany. E. Quesnel is with the Laboratoire d'Electronique de Technologie et d'Instrumentation, Département Optronique, Commissariat à l'Energie Atomique, 17 rue des Martyrs, F-38054 Grenoble Cedex 9, France.

Received 10 July 2000; revised manuscript received 16 January 2001.

0003-6935/01/132190-10\$15.00/0

(C) 2001 Optical Society of America preferred tool for specifying surface roughness. ${ }^{5}$ However, when different surfaces are to be compared, the PSD functions have to be represented together in the same graph. Hence only qualitative information can be obtained. To accomplish a quantitative microroughness description, first-order statistics such as the rms roughness or the correlation length are commonly used. ${ }^{3}$ Nevertheless, these statistics presume that the surfaces follow certain models, which in general are too simple to describe the actual properties of the surfaces. For instance, common models for representing optical surfaces are combinations of Gaussian and exponential cross-correlation functions. ${ }^{6-11}$ These models, however, cannot appropriately describe the fractal behavior. ${ }^{12}$ Moreover, Jakobs $^{13}$ showed that the behavior of the PSD of a coated sample contains two components: a fractal one caused by the substrate roughness and a second component caused by pure film roughness.

In this study we provide a means of characterizing the microroughness of optical coatings with a reduced set of parameters, each with physical meaning (such as rms roughness or correlation length). The method is based on the PSD description of a surface with subsequent extraction of characteristic parameters through fitting of the PSD to theoretical models. These models can be chosen according to the actual behavior of the PSD's.

This method has been successfully applied to a set of coatings for the VUV range. The investigation of 
these samples was driven by the technological challenges at short wavelengths: the selection of materials with low absorption and scattering in the VUV and the choice and optimization of the deposition processes. ${ }^{14,15}$ It has been proven that for applications in the UV range fluorides are the preferred materials because of their larger energy gap. However, not every deposition technique is suitable to produce appropriate fluoride coatings: Conventional evaporation techniques tend to produce coatings with high tensile stress because substrate heating is required for improving the coating adhesion and because of the large differences in thermal expansion coefficient between substrates and coating materials. However, the ion-beam-sputtering (IBS) technique solves this problem, since it does not require substrate heating, hence producing coatings with higher density and compressive stress. ${ }^{15,16}$ The roughness properties of IBS fluoride coatings are studied with the methods proposed in the present paper. In Section 2 a brief overview of the concepts involved in surface microroughness characterization is given. Then a revision of the available models to represent surface roughness is provided, and finally the method for combining these models to analyze the roughness of thin-film coatings is described. In Section 3 experimental results obtained from atomic force microscope (AFM) measurements on IBS fluoride coatings are presented, and an initial qualitative study is performed. Then the results of applying the method to the same coatings are presented. Finally, conclusions are drawn in Section 4.

\section{Measurement and Characterization of Surface Microroughness}

In this study we propose a method for reducing the large amount of data produced by microtopography measurements. Although the measurements presented in this study were performed with an AFM, the method is suitable for any technique that results in the surface profile of the sample as a one- or twodimensional function of the position such as mechanical profilometry, white-light interferometry, or confocal microscopy.

The simplest reduction procedure consists of characterizing the surface by its $\mathrm{rms}$ roughness $\left(\sigma_{\mathrm{rms}}\right){ }^{3}$ This quantity is useful for comparing different surfaces. It is well known, however, that the rms roughness depends on the spatial-frequency bandwidth of the particular measurement technique. Therefore, when the results from different measurements are compared, the measurement conditions (scan length and sampling distance) need to be equivalent. Moreover, the rms roughness does not give information about the spatial distribution; this means that two surfaces with the same rms roughness could contain different lateral structures.

The PSD yields a more complete roughness description, since it takes into account the statistical correlation between the different surface points. It is defined as the Fourier transform of the surface autocovariance function. ${ }^{17}$ In optical coating tech-

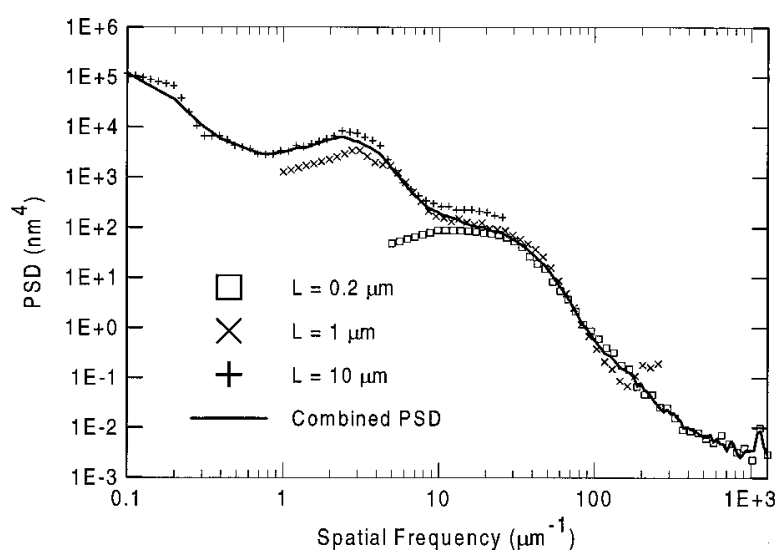

Fig. 1. Example of the combination of PSD functions corresponding to different scan sizes.

nology it is common to use the two-dimensional isotropic form of the PSD, since the majority of optical coatings and their substrates justify sufficiently the assumption of isotropic roughness. This PSD is then related to the surface autocovariance function through the Hankel transform. ${ }^{6}$

PSD functions obtained from measurements are defined only in a limited range of spatial frequencies. This range depends on the scan length and the sampling distance, and it also can be additionally reduced by the effect of measurement artifacts. ${ }^{13,18}$ These limitations can be overcome when we combine measurements performed on different scales and/or with different measuring methods. ${ }^{19}$ These measurements should fulfill at least two conditions:

(i) The spatial-frequency ranges where the measurements are defined should partially overlap. This condition is easy to meet by adequate selection of the scan sizes and sampling distances.

(ii) In the overlapping region the different PSD functions should be of the same order of magnitude.

Then the result of the PSD combination is a function whose value at a given frequency $f$ is the geometrical average over all the PSD's that are defined at this frequency,

$$
\operatorname{PSD}_{\text {combined }}(f)=\left[\prod_{i=1}^{N} \operatorname{PSD}_{i}(f)\right]^{1 / N},
$$

where $N$ is the number of PSD functions overlapping at that particular frequency.

Figure 1 shows an example of this procedure: The original PSD functions correspond to AFM measurements of a sample corresponding to a three-layer fluoride coating on $\mathrm{CaF}_{2}$. A more complete description of all studied samples is given below. The measurements were performed at the same position and with three different scan sizes: $0.2 \mu \mathrm{m} \times 0.2 \mu \mathrm{m}, 1$ $\mu \mathrm{m} \times 1$ and $10 \mu \mathrm{m} \times 10 \mu \mathrm{m}$. The decrease observed at the low-frequency range in the original PSD functions is a result of the detrending procedure commonly applied to the raw data. ${ }^{12}$ These points are 
not considered for calculating the combined PSD. Furthermore, the influence of noise can be perceived in the high-spatial-frequency ranges of each individual measured PSD. These points have not been considered for the combination, either. With this example it is shown that this procedure enables the generation of a complete description of surface roughness on a large bandwidth of spatial frequencies. This procedure can be applied to combine any number of microtopography measurements, including measurements taken at different positions on the same sample or even measurements performed with different methods.

Although the PSD has a more complete description of the surface than the rms roughness, it is difficult to compare the roughness characteristics of different samples on the basis of a function. Consequently, it is useful to obtain quantitative information from the PSD's that can be used to make the comparison between samples easier. This quantitative information can be extracted with the help of mathematical models.

\section{A. Overview of Models Applied in Surface Characterization}

PSD functions contain a complete description of the surfaces' statistics in the sense that they do not only take into account the height statistics but also contain information on the surface lateral details. A further step in the PSD interpretation is to model them as analytical functions that depend on a reduced set of characteristic parameters. Provided that these parameters possess a physical meaning, their value is of use to characterize the surface. In this section the mathematical models available for the representation of PSD functions and their relation to the types of surfaces commonly found in optical coating technology are presented.

Several statistical models have been used in the past for roughness description of optical surfaces and thin films. These models consist of a function or combination of functions approximating the experimental PSD behavior. The most extended model for the PSD of a coating is the sum of Hankel transforms of a Gaussian and an exponential autocorrelation function. ${ }^{6-11,20}$ The corresponding PSD's have the form

$$
\begin{aligned}
\operatorname{PSD}_{\text {gauss }}\left(f ; \sigma_{s}, \tau_{s}\right) & =\pi \sigma_{s}{ }^{2} \tau_{s}^{2} \exp \left(-\pi^{2} \tau_{s}^{2} f^{2}\right), \\
\operatorname{PSD}_{\text {exp }}\left(f ; \sigma_{l}, \tau_{l}\right) & =\frac{2 \pi \sigma_{l}^{2} \tau_{l}^{2}}{\left(1+4 \pi^{2} \tau_{l}^{2} f^{2}\right)^{3 / 2}} .
\end{aligned}
$$

In this model the characteristic parameters are the surface rms roughnesses $\sigma$ and the correlation lengths $\tau$ for both the Gaussian and the exponential functions. The $\sigma$ and the $\tau$ are the result of applying integral expressions (4) and (5) of Ref. 12 for all the spatial frequencies from 0 to infinity. This means that these rms roughnesses and correlation lengths become independent of the scale, and thus they are suitable for comparing different surfaces. The cor- relation length $\tau$ is a measure of the characteristic lateral size of the structures on the surface. As a result of the fast decrease of the Gaussian function, it is adequate for surface structures with short-range correlation length ( $s$ subscript). However, the exponential model has a much slower decrease, thus making it suitable for long-correlated surface structures $(l$ subscript).

This model has been extensively used and has led to satisfactory results. However, it is not completely correct when a wide range of spatial frequencies are considered. It has been demonstrated by AFM and scattering measurements ${ }^{13}$ that the PSD of a coating can be actually approximated by the sum of the PSD of the substrate and the PSD of the pure thin film. The PSD's of the substrates follow an inverse power law that cannot be approximated by either the Gaussian or the exponential PSD's. To this end the fractal $^{12,17}$ model is used to describe these roughnesses,

$$
\operatorname{PSD}_{\text {fractal }}(f ; K, n)=K / f^{n+1},
$$

where $K$ has units of length to the power of $(3-n)$. Here rms roughness and correlation length cannot be defined for the fractal model, and consequently the parameters $K$ and $n$ are the values taken to characterize the surface. This PSD form is obtained when it is assumed that the surface is self-affine. ${ }^{21}$ This is a common characteristic of highly finished optical surfaces.

An alternative function for describing the PSD of the pure thin film is the $A B C$ model $^{21,22}$ (also called the $k$-correlation model in some references):

$$
\operatorname{PSD}_{A B C}(f ; A, B, C)=\frac{A}{\left(1+B^{2} f^{2}\right)^{(C+1) / 2}},
$$

where $C$ is a constant greater than 2 . Unlike the previous models, the $A B C$ model does not correspond to any defined autocovariance function. Nevertheless, specific information about the surface microstructure can be extracted from the parameters $A, B$, and $C$. Following Eqs. (4) and (5) of Ref. 12, equivalent rms roughnesses and correlation lengths that depend on the three parameters can be derived:

$$
\sigma_{\mathrm{ABC}}^{2}=\frac{2 \pi A}{B^{2}(C-1)}, \quad \tau_{\mathrm{ABC}}{ }^{2}=\frac{(C-1)^{2} B^{2}}{2 \pi^{2} C} .
$$

From analyzing expressions (5) and (6) it can be deduced that the parameter $C$ provides the possibility of having a continuous transition model between the exponential $(C=2)$ and the gaussian $(C \gg 2)$ models.

All models presented up to this point are monotonically decreasing functions of the spatial frequency. However, it has been observed that several coatings show the formation of superstructures uniformly distributed along the surface. ${ }^{23,24}$ This induces a local maximum in the lower frequencies of the PSD that cannot be described satisfactorily by any of the previous models. An example is represented by the PSD of Fig. 1, where the local maximum can be recognized at a frequency near $3 \mu \mathrm{m}^{-1}$. A convenient 
PSD for the modeling of this local maximum is a Gaussian function with its maximum shifted to a nonzero spatial frequency ${ }^{25}$ :

$$
\begin{aligned}
\operatorname{PSD}_{\mathrm{sh}}\left(f ; \sigma_{\mathrm{sh}}, \tau_{\mathrm{sh}}, f_{\mathrm{sh}}\right)= & \pi \sigma_{\mathrm{sh}}{ }^{2} \tau_{\mathrm{sh}}{ }^{2} \exp \left[-\pi^{2} \tau_{\mathrm{sh}}{ }^{2}(f\right. \\
& \left.\left.-f_{\mathrm{sh}}\right)^{2}\right] .
\end{aligned}
$$

This PSD corresponds to an autocovariance function with the form of a Gaussian multiplied by a cosine. The period of the cosine is related to the periodicity of the superstructures in the surface. This period is translated into the spatial-frequency domain as the shift of the PSD maximum to the frequency $f_{\mathrm{sh}}$. The meaning of the other model parameters can also be related to other characteristics of these superstructures: The $\tau_{\mathrm{sh}}$ corresponds to their size; $\sigma_{\mathrm{sh}}$, to their height.

So far, no combinations of these models have been used for a quantitative surface-roughness description, even when the experimentally obtained PSD's suggested that such a combination would be useful. The reason might have been the lack of a method indicating the steps to find the correct combination and providing prescriptions for extracting meaningful values. In this study we suggest such a method, which is based on the use of the models outlined above.

\section{B. Analysis Method}

The surface-roughness analysis of a given optical coating or substrate is accomplished by means of fitting the experimentally obtained PSD to a combination of models. This combination (and thus the number of parameters that characterize the surface) will depend on each particular sample. The combination has to be chosen through examination of the experimental PSD and recognition of the different roughness contributions.

In this study, for instance, we use the following particular combinations. For the substrates the fractal model is applied,

$$
\operatorname{PSD}_{\text {substrate }}(f ; K, n)=\operatorname{PSD}_{\text {fractal }}(f ; K, n),
$$

where only two parameters are needed for the description. A convenient model for a large variety of coatings is the combination of the fractal and the $A B C$ models:

$$
\begin{array}{r}
\operatorname{PSD}_{\text {coat1 } 1}\left(f ; K, n, \sigma_{A B C}, \tau_{A B C}, C\right)=\operatorname{PSD}_{\text {fractal }}(f ; K, n) \\
+\operatorname{PSD}_{A B C}\left(f ; \sigma_{A B C}, \tau_{A B C}, C\right) .
\end{array}
$$

The fractal function takes into account the substrate influence, whereas the $A B C$ model is directly related to the intrinsic roughness of the coating. The $A B C$ model has been found to be more adequate than the Gaussian, the exponential, or their sum, because the exponent $C$ enables better matching of the film PSD. This model needs five parameters to describe the surface $\left(K, n, \sigma_{A B C}, \tau_{A B C}\right.$, and $\left.C\right)$. Finally, as is demonstrated in detail in Subsection 3.B, a more elaborated model is needed to fit the PSD of coatings showing the superstructures mentioned above. In this case the sum of three PSD functions is considered:

$$
\begin{aligned}
& \operatorname{PSD}_{\text {coat } 2}\left(f ; K, n, \sigma_{A B C}, \tau_{A B C}, C, \sigma_{\mathrm{sh}}, \tau_{\mathrm{sh}}, f_{\mathrm{sh}}\right) \\
&=\operatorname{PSD}_{\text {fractal }}(f ; K, n)+\operatorname{PSD}_{A B C}\left(f ; \sigma_{A B C}, \tau_{A B C}, C\right) \\
&+\operatorname{PSD}_{\mathrm{sh}}\left(f ; \sigma_{\mathrm{sh}}, \tau_{\mathrm{sh}}, f_{\mathrm{sh}}\right),
\end{aligned}
$$

where as many as seven parameters are necessary.

The fitting procedure is carried out by minimization of a merit function that is a measure of the difference between the experimental PSD and the theoretical model. Thus if $\mathbf{P}$ is the set of parameters that characterize the model under consideration, the merit function is defined as

$$
\begin{aligned}
\operatorname{Merit}(\mathbf{P})= & \frac{1}{N} \sum_{i=1}^{N}\left[\log \operatorname{PSD}_{\text {measured }}\left(f_{i}\right)\right. \\
& \left.-\log \operatorname{PSD}_{\text {model }}\left(f_{i} ; \mathbf{P}\right)\right]^{2} .
\end{aligned}
$$

In this expression the $f_{i}$ are the spatial frequencies at which the measured PSD is evaluated and $N$ is the number of points at which the PSD is sampled. This merit function is a customized form of the chi-square function, widely used in the fitting of experimental data. ${ }^{26}$ The customization consists of substracting the logarithms of the PSD's instead of the PSD's themselves. This is necessary because the values of the PSD function can extend over several orders of magnitude and because a direct comparison would give more weight to the spatial frequencies where the PSD value is higher. This form of merit function has been successfully applied to the fitting of angleresolved scattering measurements. ${ }^{27}$

A downhill simplex algorithm ${ }^{26,28}$ was applied to accomplish the merit function minimization. The result of this minimization is a parameter set $\mathbf{P}$ providing the best fit of the model to the experimental data. The values of these parameters are then taken as the surface characteristic values.

The main advantage of this method is that it can be adapted easily to any kind of surface, regardless of its particular characteristics, by means of choosing the combination of PSD models that matches the exper-

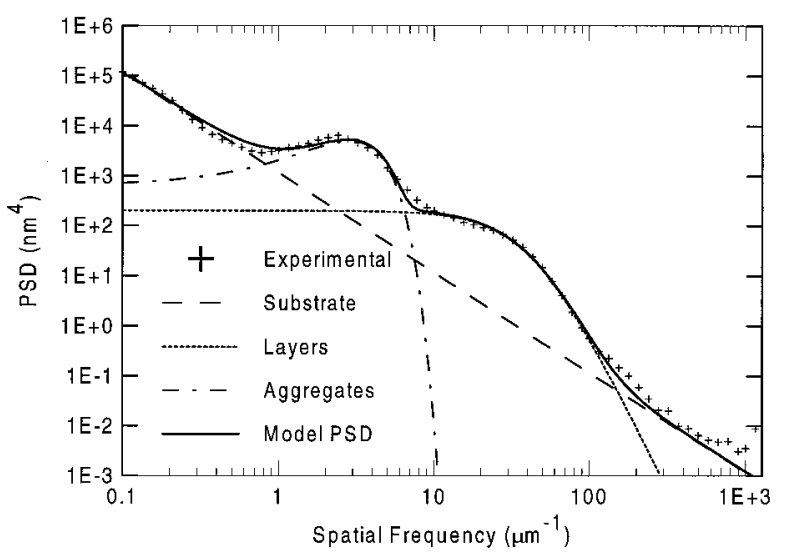

Fig. 2. Example of fitting of a measured PSD to a theoretical model. See text for the details. 
Table 1. Result of the Roughness Modeling of Sample $\mathrm{S} / \mathrm{L} / \mathrm{H} / \mathrm{L}$ on $\mathrm{CaF}_{2}$

\begin{tabular}{|c|c|c|c|}
\hline Component & Substrate & Layers & Aggregates \\
\hline Model & Fractal & $A B C$ & Shifted Gaussian \\
\hline Characteristic parameters & $\begin{aligned} K & =1150.00 \mathrm{~nm}^{3-n} \\
n & =1.00\end{aligned}$ & $\begin{aligned} \sigma_{A B C} & =0.73 \mathrm{~nm} \\
\tau_{A B C} & =3.68 \mathrm{~nm} \\
C & =5.43\end{aligned}$ & $\begin{array}{l}\sigma_{s}=0.19 \mathrm{~nm} \\
\tau_{s}=173.10 \mathrm{~nm} \\
f_{s}=2.86 \mu \mathrm{m}^{-1}\end{array}$ \\
\hline
\end{tabular}

imental PSD behavior without making any a priori assumption.

As an example of this analysis method we present in Fig. 2 the roughness characterization of the sample corresponding to the PSD presented in Fig. 1. The pluses represent the PSD function resulting from the combination of the different measurements. The model components are represented separately as discontinuous lines. The straight dashed line corresponds to the fractal PSD modeling the substrate. The dotted curve is the $A B C$ PSD used to represent the intrinsic film roughness. Finally, the dasheddotted curve is a shifted Gaussian taking into account the influence of the superstructures. The thick continuous curve is the sum of the three functions: the best fit obtained for these specific measured data. The corresponding parameters characterizing the surface roughness are presented in Table 1.

In summary, the method consists of the following steps:

(i) Calculate the PSD from each measurement and combine all the PSD functions into a single one, with the combination procedure.

(ii) Choose the appropriate combination of theoretical PSD functions that is likely to best match the PSD behavior.

(iii) Fit this combination to the experimental PSD to obtain the values that characterize the sample.

\section{Experimental Results}

A. Atomic Force Microscope Measurements and Calculation of Experimental Power Spectral Densities

The method described in Subsection 2.B was applied to a set of samples consisting of single-layer and mul- tilayer fluoride coatings deposited by IBS. The experiment was intended to study the elastic lightscattering properties in the VUV spectral region and the relation of these properties to the film microstructure. The results of this study have been published elsewhere. ${ }^{23,24}$ Table 2 summarizes the produced designs, where the thickness units are optical quarter-waves and the design wavelength is $193 \mathrm{~nm}$. $\mathrm{MgF}_{2}$ and $\mathrm{LaF}_{3}$ were used as the low- and high-index materials, respectively. In the table, $\mathrm{L}(\mathrm{H})$ stands for a quarter-wave optical thickness layer of lowindex (high-index) material. The substrates used were superpolished UV-grade fused silica (FS) and $\mathrm{CaF}_{2}$. The designs were chosen to include the coating thickness (or equivalently, the number of layers) as variables in the study.

The microroughness was measured with an AFM (Digital Instruments, Model dimension 3000) operated in the proprietary Tapping Mode. Silicon tips with $10-\mathrm{nm}$ radius were employed. The measurements consisted of matrices of $512 \times 512$ points with scan sizes of $0.2 \mu \mathrm{m} \times 0.2 \mu \mathrm{m}$ (on some selected samples), $1 \mu \mathrm{m} \times 1 \mu \mathrm{m}$, and $10 \mu \mathrm{m} \times 10 \mu \mathrm{m}$. Figures 3 and 4 are examples of two of these measurements. Figure 3 corresponds to the $\mathrm{S} / \mathrm{L}$ design onto $\mathrm{CaF}_{2}$. A granular structure related to the tops of the columnar structures, which are typical for fluoride coatings, can be observed in the left-hand image $(\mathrm{L}=$ $1 \mu \mathrm{m})$. Figure 4 corresponds to the $\mathrm{S} / \mathrm{L} / \mathrm{H} / \mathrm{L}$ design onto $\mathrm{CaF}_{2}$ and shows a particular phenomenon of IBS fluoride coatings that was observed on some samples: The columnar structures revealed a tendency to aggregate and form superstructures uniformly distributed, resulting in an increase in rms roughness. This tendency to aggregate is a particular character-

Table 2. Designs of the Samples Studied in This Paper

\begin{tabular}{|c|c|c|c|c|}
\hline \multirow{2}{*}{$\begin{array}{l}\text { Optical Thickness } \\
(\mathrm{QWOT})^{a}\end{array}$} & \multicolumn{2}{|c|}{ FS Substrate } & \multicolumn{2}{|c|}{$\mathrm{CaF}_{2}$ Substrate } \\
\hline & $\mathrm{QW}^{b}$ Designs & $\mathrm{HW}^{c}$ Designs & QW Designs & HW Designs \\
\hline 1 & $\mathrm{~S} / \mathrm{L}$ & & $\mathrm{S} / \mathrm{L}, \mathrm{S} / \mathrm{H}$ & \\
\hline 2 & $\mathrm{~S} / \mathrm{L} / \mathrm{H}$ & $\mathrm{S} / 2 \mathrm{~L}$ & $\mathrm{~S} / \mathrm{L} / \mathrm{H}$ & $\mathrm{S} / 2 \mathrm{H}$ \\
\hline 3 & $\mathrm{~S} / \mathrm{L} / \mathrm{H} / \mathrm{L}$ & & $\mathrm{S} / \mathrm{L} / \mathrm{H} / \mathrm{L}$ & \\
\hline 4 & & & & $\mathrm{~S} / 2 \mathrm{~L} / 2 \mathrm{H}$ \\
\hline 6 & $\mathrm{~S} /(\mathrm{L} / \mathrm{H})^{3}$ & $\mathrm{~S} / 2 \mathrm{~L} / 2 \mathrm{H} / 2 \mathrm{~L}$ & & $\mathrm{~S} / 2 \mathrm{~L} / 2 \mathrm{H} / 2 \mathrm{~L}$ \\
\hline 7 & $\mathrm{~S} /(\mathrm{L} / \mathrm{H})^{3} / \mathrm{L}$ & & & \\
\hline 10 & $\mathrm{~S} /(\mathrm{L} / \mathrm{H})^{5}$ & $\mathrm{~S} / 2 \mathrm{~L} / 2 \mathrm{H} / 2 \mathrm{~L} / 2 \mathrm{H} / 2 \mathrm{~L}$ & & \\
\hline 20 & $\mathrm{~S} /(\mathrm{L} / \mathrm{H})^{10}$ & & $\mathrm{~S} /(\mathrm{L} / \mathrm{H})^{10}$ & $\mathrm{~S} /(2 \mathrm{~L} / 2 \mathrm{H})^{5}$ \\
\hline
\end{tabular}

${ }^{a}$ Quarter-wave optical thickness layer.

${ }^{b}$ Quarter-wave.

${ }^{c}$ Half-wave. 


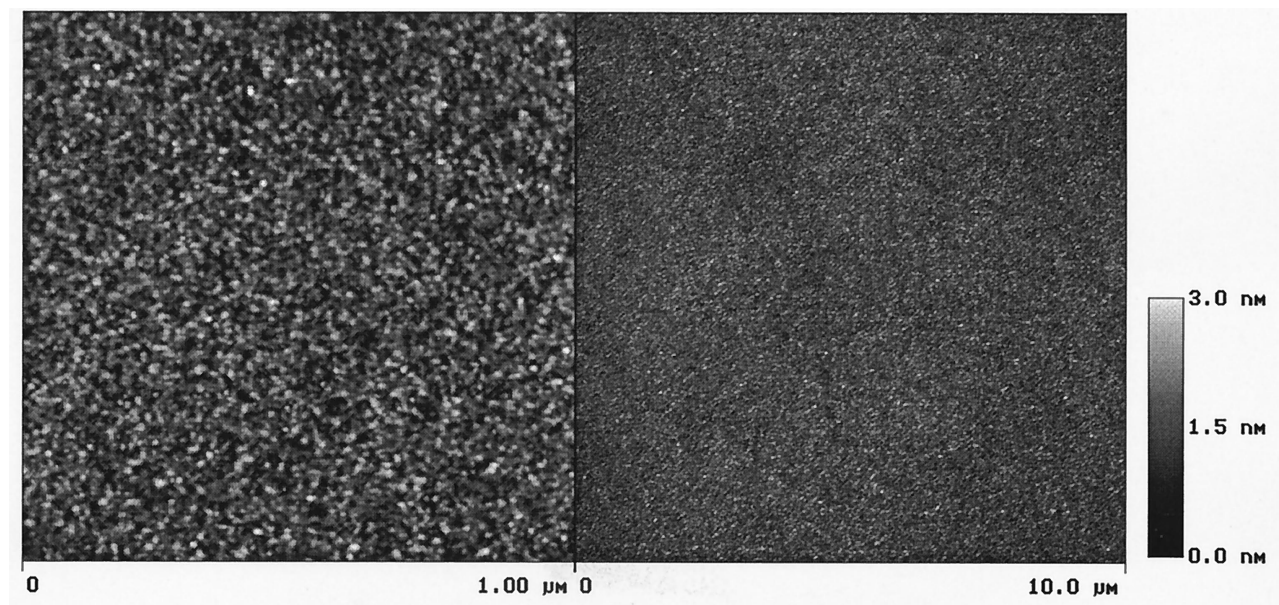

Fig. 3. Top view $\mathrm{AFM}$ image of sample $\mathrm{S} / \mathrm{L}$ on $\mathrm{CaF}_{2}$.

istic of the fluoride coatings studied in this paper and is not commonly observed in other types of coating. For this reason we will distinguish throughout this paper between the roughness induced by these aggregates and the roughness induced by the granular structure of the coating (which will be called the intrinsic roughness of the coating). Figure 5 displays a selection of the PSD functions for the designs with quarter-wave layers. Graph (a) represents the data for the samples deposited on FS, and graph (b) corresponds to the $\mathrm{CaF}_{2}$ substrate. The PSD's of the bare substrates have been included as a reference.

First we observe that the PSD's of both substrates follow the expected inverse power law of the fractal model with the exception of the lower frequencies where the scanner bow effect ${ }^{13}$ can give rise to an upward deviation. The PSD functions of quarterwave single-layer designs reveal a roughness component related to the fluoride columnar structure observed at high spatial frequencies. In the lowspatial-frequency range the roughness statistics of the substrate is reproduced by the films.

The PSD of the $\mathrm{S} / \mathrm{L} / \mathrm{H}$ coating is similar to that of the $\mathrm{S} / \mathrm{L}$ sample for both substrates. This indicates that the high-index quarter-wave layer adds a small roughness component. The addition of a second lowindex quarter-wave layer induces the formation of aggregates as seen in Fig. 4. These aggregates lead to the appearance of a local maximum in the PSD near $3 \mu \mathrm{m}^{-1}$. By observing the PSD of the $\mathrm{S} /(\mathrm{L} / \mathrm{H})^{10}$ samples, where the local maximum is overridden by the intrinsic roughness component of the coating, it can be concluded that the aggregates' height does not vary when new layers are added. To illustrate this fact, Fig. 6 presents the measurement of sample $\mathrm{S} /(\mathrm{L} / \mathrm{H})^{10}$ onto $\mathrm{CaF}_{2}$ : the aggregates are still present but with a much lower contrast as compared with Fig. 4, a fact that makes it difficult to distinguish them from the coating roughness.

To complete this presentation of experimental results, a selection of the PSD functions for the halfwave designs is presented in Fig. 7. As in the previous case, the PSD of the substrate has been included for reference. The presence of aggregates can be observed in samples $\mathrm{S} / 2 \mathrm{~L} / 2 \mathrm{H} / 2 \mathrm{~L}$ and $\mathrm{S} / 2 \mathrm{~L} / 2 \mathrm{H} / 2 \mathrm{~L} / 2 \mathrm{H} / 2 \mathrm{~L}$ on $\mathrm{FS}$ and $\mathrm{S} / 2 \mathrm{~L} / 2 \mathrm{H}$ on $\mathrm{CaF}_{2}$.

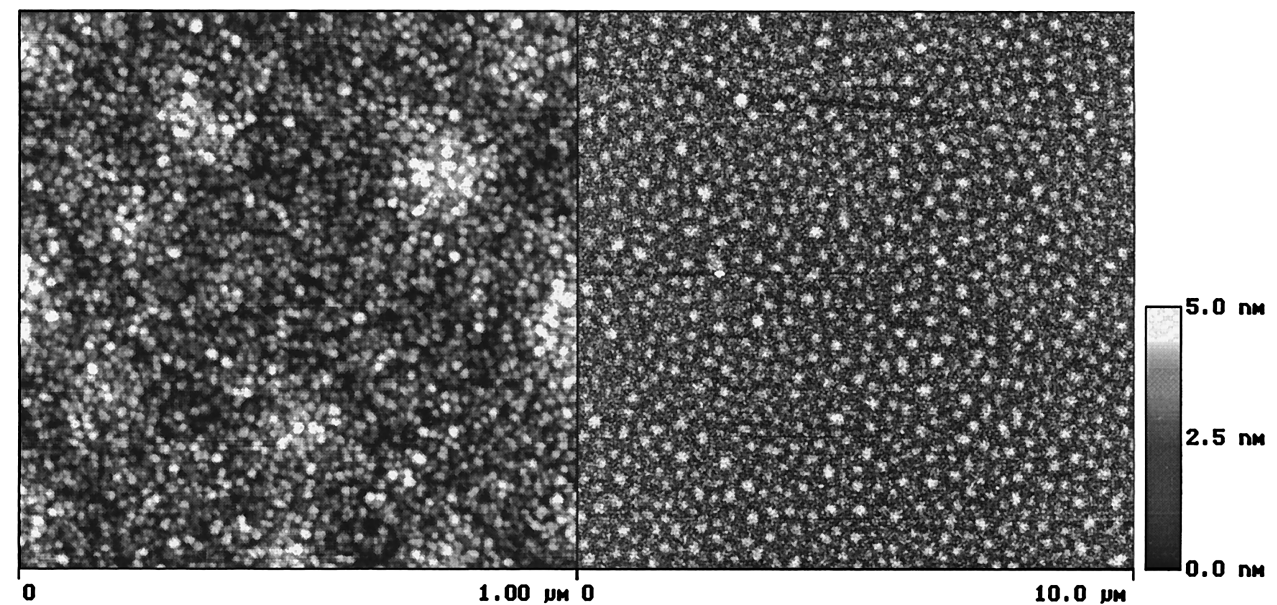

Fig. 4. Top view $A F M$ image of sample $\mathrm{S} / \mathrm{L} / \mathrm{H} / \mathrm{L}$ on $\mathrm{CaF}_{2}$. 


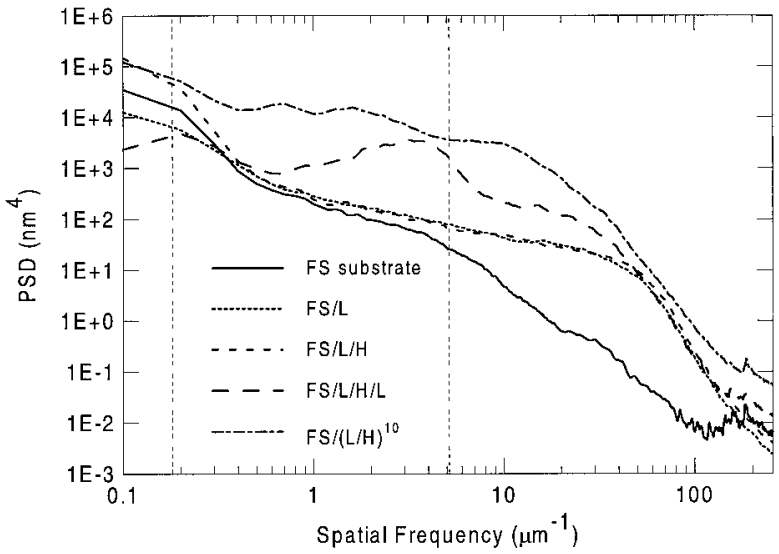

(a)

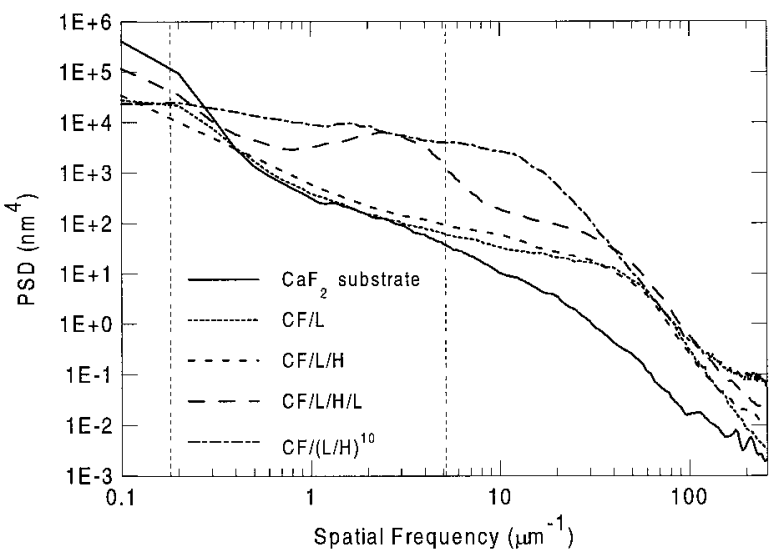

(b)

Fig. 5. Selected PSD functions of the samples with quarter-wave stacks. (a) FS substrate. (b) $\mathrm{CaF}_{2}$ substrate.

\section{B. Microroughness Analysis of Ultraviolet Coatings}

The analysis method presented above has been used for a complete roughness characterization of the samples described in Subsection 3.A. Each PSD has been fitted to the suitable model; this includes a fractal for the substrates, a fractal and an $A B C$-PSD for

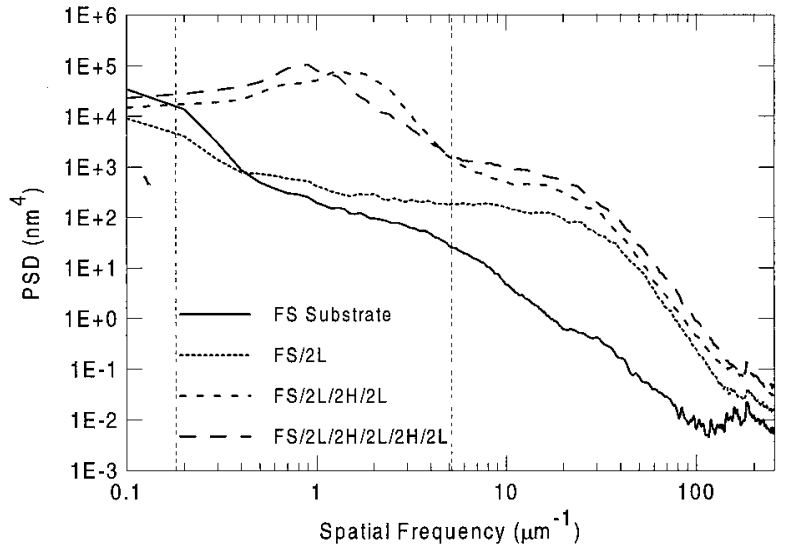

(a)

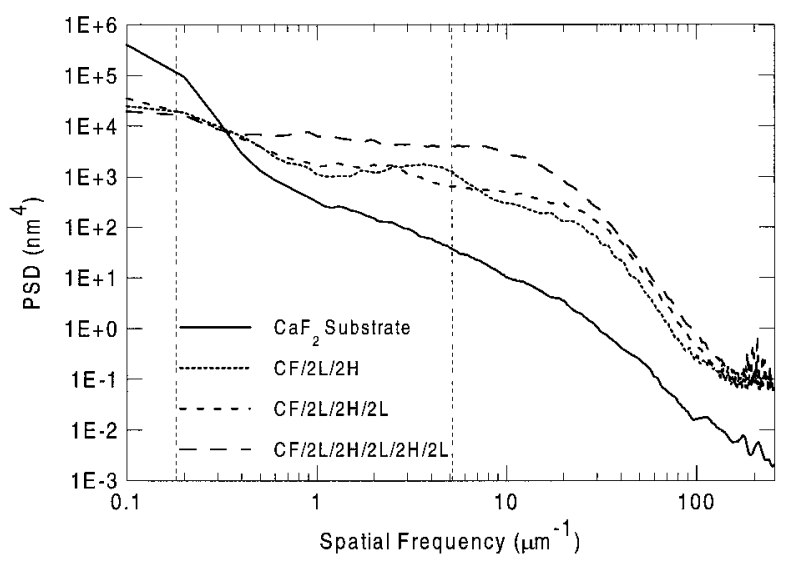

(b)

Fig. 7. Selected PSD functions of the samples with coatings consisting of half-wave layer stacks. (a) FS substrate. (b) $\mathrm{CaF}_{2}$ substrate.

the samples without aggregates, and finally a fractal plus an $A B C$ plus a shifted Gaussian for the rest of samples.

The results are summarized in Tables 3 and 4 . The tables contain the resulting rms roughnesses and

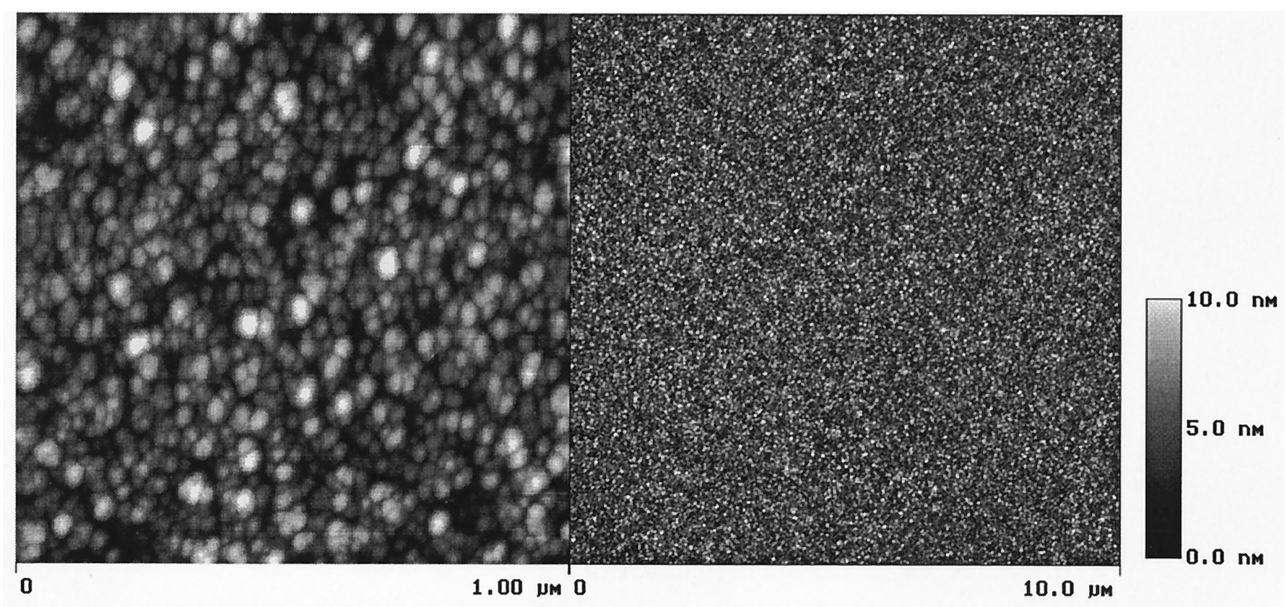

Fig. 6. Top view AFM image measurement of sample $\mathrm{S} /(\mathrm{L} / \mathrm{H})^{10}$ on $\mathrm{CaF}_{2}$. 
Table 3. Roughness Characterization of the Coatings Deposited on FS

\begin{tabular}{|c|c|c|c|c|c|c|}
\hline \multirow[b]{3}{*}{ Design } & \multicolumn{6}{|c|}{ Model } \\
\hline & \multicolumn{3}{|c|}{$A B C$} & \multicolumn{3}{|c|}{ Shifted Gaussian } \\
\hline & $\sigma_{A B C}(\mathrm{~nm})$ & $\tau_{A B C}(\mathrm{~nm})$ & $C$ & $\sigma_{s}(\mathrm{~nm})$ & $\tau_{s}(\mathrm{~nm})$ & $f_{s}\left(\mu \mathrm{m}^{-1}\right)$ \\
\hline $\mathrm{S} / \mathrm{L}$ & 0.45 & 9.35 & 6.41 & & & \\
\hline $\mathrm{S} / \mathrm{L} / \mathrm{H}$ & 0.47 & 8.37 & 7.20 & & & \\
\hline $\mathrm{S} / \mathrm{L} / \mathrm{H} / \mathrm{L}$ & 0.72 & 11.66 & 9.10 & 0.19 & 173.10 & 3.45 \\
\hline $\mathrm{S} /(\mathrm{L} / \mathrm{H})^{3}$ & 1.05 & 11.66 & 6.43 & 0.20 & 260.60 & 1.93 \\
\hline $\mathrm{S} /(\mathrm{L} / \mathrm{H})^{3} \mathrm{~L}$ & 1.13 & 13.14 & 6.87 & 0.23 & 186.68 & 2.32 \\
\hline $\mathrm{S} /(\mathrm{L} / \mathrm{H})^{5}$ & 1.34 & 13.42 & 6.13 & 0.19 & 226.06 & 1.70 \\
\hline $\mathrm{S} /(\mathrm{L} / \mathrm{H})^{10}$ & 1.81 & 18.60 & 4.81 & 0.22 & 237.14 & 1.53 \\
\hline $\mathrm{S} / 2 \mathrm{~L}$ & 0.66 & 10.98 & 8.06 & & & \\
\hline $\mathrm{S} / 2 \mathrm{~L} / 2 \mathrm{H}$ & 1.12 & 14.96 & 4.14 & 0.53 & 270.09 & 1.64 \\
\hline $\mathrm{S} / 2 \mathrm{~L} / 2 \mathrm{H} / 2 \mathrm{~L} / 2 \mathrm{H} / 2 \mathrm{~L}$ & 1.52 & 14.84 & 4.12 & 0.42 & 331.29 & 1.07 \\
\hline
\end{tabular}

correlation lengths corresponding to the $A B C$ model as well as the value of the exponent $C$. For the samples with aggregates the shifted-Gaussian characteristic parameters are included as well. The $K$ and the $n$ values obtained from the characterization of the samples were similar to those obtained for the bare substrates. This is a proof of the consistence of the analysis method, since it is able to separate correctly the substrate roughness component from the total roughness of a coated sample. Consequently, only the $K$ and the $n$ values for the substrates are presented in Table 5.

The results of the method allow for access to observations that would not be possible from simple PSD examination. First, a comparison of the growth of film roughness with thickness depending on the substrate type can be performed. This comparison is presented in Figs. 8 and 9. In Fig. 8 the intrinsic rms roughness of the coating (resulting from the $A B C$ component) is represented for the quarter-wave samples. It can be seen that the roughness grows with coating thickness and that this growth is not influenced by the substrate, since the values are similar for both substrates. It is important to note that the high-index quarter-wave layer adds a smaller com- ponent of roughness than the low-index one. This can be seen when we compare the values for the samples $\mathrm{S} / \mathrm{L}$ and $\mathrm{S} / \mathrm{L} / \mathrm{H}$. This small increase is related to the combination of two effects: On one side, the physical thickness of a high-index layer is smaller. On the other side, the $\mathrm{LaF}_{3}$ films show a globular structure in opposition to the columnar structure of the $\mathrm{MgF}_{2}$ films. This globular structure seems to promote a lower roughness.

From Fig. 8 it can also be observed that the rms roughness increases with physical thickness following a power law. More precisely, if $T$ is the physical thickness of the coating, the intrinsic rms roughness $(\sigma)$ follows $\sigma(T) \propto T^{\beta}$, with $\beta=0.52$ for the samples with FS substrates and $\beta=0.51$ for those with $\mathrm{CaF}_{2}$ substrates. This result has to be taken as an average for both $\mathrm{MgF}_{2}$ and $\mathrm{LaF}_{3}$ materials. Furthermore, these values are in good agreement with the results obtained from total scattering measurements for $\mathrm{PbF}_{2}$ coatings deposited by plasma chemical vapor deposition. ${ }^{29}$

The correlation length of the quarter-wave samples is presented in Fig. 9. Again, the behavior of this parameter is similar for the samples on FS and for the $\mathrm{CaF}_{2}$ substrates: a gradual increase with total

Table 4. Roughness Characterization of the Coatings Deposited on $\mathrm{CaF}_{2}$

\begin{tabular}{|c|c|c|c|c|c|c|}
\hline \multirow[b]{3}{*}{ Design } & \multicolumn{6}{|c|}{ Model } \\
\hline & \multicolumn{3}{|c|}{$A B C$} & \multicolumn{3}{|c|}{ Shifted Gaussian } \\
\hline & $\sigma_{A B C}(\mathrm{~nm})$ & $\tau_{A B C}(\mathrm{~nm})$ & $C$ & $\sigma_{s}(\mathrm{~nm})$ & $\tau_{s}(\mathrm{~nm})$ & $f_{s}\left(\mu \mathrm{m}^{-1}\right)$ \\
\hline $\mathrm{S} / \mathrm{L}$ & 0.42 & 7.58 & 7.25 & & & \\
\hline $\mathrm{S} / \mathrm{L} / \mathrm{H}$ & 0.43 & 9.10 & 5.77 & & & \\
\hline $\mathrm{S} / \mathrm{L} / \mathrm{H} / \mathrm{L}$ & 0.73 & 9.88 & 5.43 & 0.25 & 160.22 & 2.86 \\
\hline $\mathrm{S} /(\mathrm{L} / \mathrm{H})^{10}$ & 1.71 & 21.18 & 5.27 & 0.21 & 170.96 & 0.60 \\
\hline $\mathrm{S} / 2 \mathrm{~L} / 2 \mathrm{H}$ & 0.75 & 13.11 & 9.16 & 0.14 & 152.17 & 3.97 \\
\hline $\mathrm{S} / 2 \mathrm{~L} / 2 \mathrm{H} / 2 \mathrm{~L}$ & 1.05 & 12.40 & 7.66 & 0.06 & 253.30 & 2.23 \\
\hline $\mathrm{S} /(2 \mathrm{~L} / 2 \mathrm{H})^{5}$ & 1.96 & 17.92 & 5.19 & 0.02 & 1126.04 & 0.94 \\
\hline $\mathrm{S} / \mathrm{H}$ & 0.45 & 22.54 & 3.32 & & & \\
\hline $\mathrm{S} / 2 \mathrm{H}$ & 0.24 & 39.89 & 7.06 & 0.24 & 169.95 & 2.11 \\
\hline
\end{tabular}


Table 5. Roughness Characterization of the Substrates

\begin{tabular}{ccc}
\hline Substrate & $K\left(\mathrm{~nm}^{3-n}\right)$ & $n$ \\
\hline $\mathrm{FS}$ & 337.30 & 1.05 \\
$\mathrm{CaF}_{2}$ & 980.31 & 1.24 \\
\hline
\end{tabular}

coating thickness. However, the establishment of a model for this behavior is not as clear as in the case of the rms roughness.

The analysis procedure presented here enables us to extract information about the aggregates as well: In the shifted-Gaussian model the aggregate size is characterized by the parameter $\tau_{\mathrm{sh}}$; their periodicity is related to the central frequency $f_{\text {sh }}$; and, finally, their height is given by $\sigma_{\mathrm{sh}}$. As stated above in the qualitative description of the experimental results of Section 3, the height of the aggregates remains at the same level when the coating thickness increases. This qualitative information can now be quantitatively confirmed: The values of $\sigma_{\mathrm{sh}}$ for the quarterwave samples are all in the range of $0.19-0.25 \mathrm{~nm}$ and do not show any clear tendency of increase with thickness. Two further conclusions can be extracted from the characteristic parameters of the aggregates: First, their size tends to increase slowly with the coating thickness; and second, the center frequency decreases slowly with thickness, indicating that periodicity of the aggregates on the surface increases.

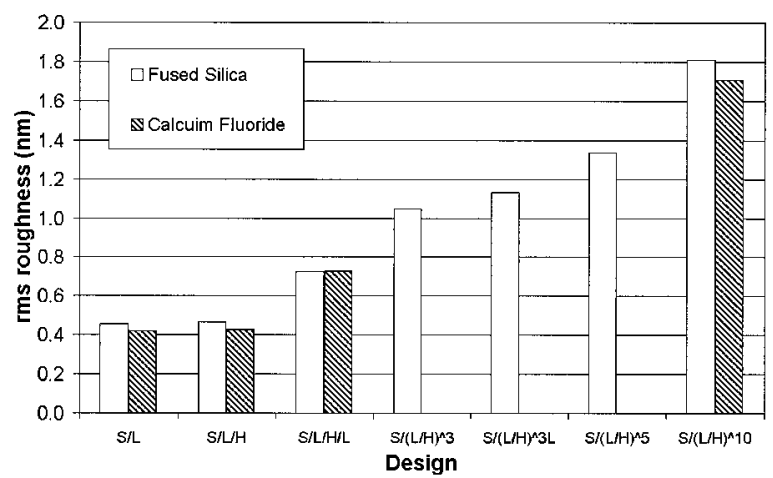

Fig. 8. Intrinsic rms roughness of the coatings with quarter-wave designs.

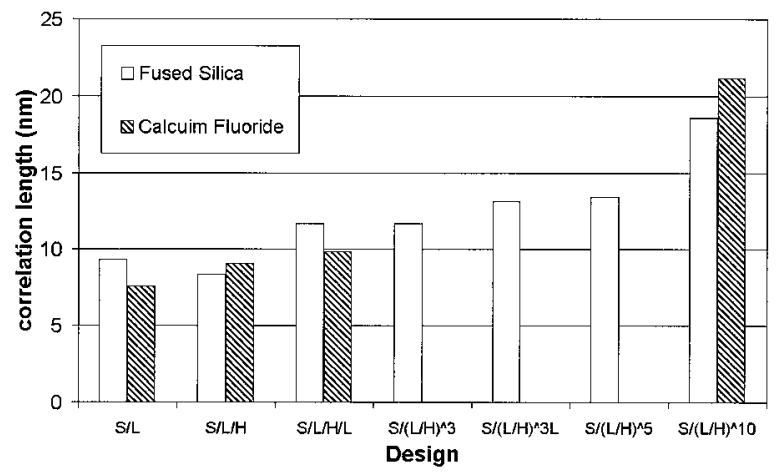

Fig. 9. Intrinsic correlation length of the coatings with quarterwave designs.

\section{Conclusions}

In this study a method for comprehensive analysis of the microroughness of optical surfaces and coatings has been presented. The method is capable of extracting a reduced set of parameters that characterize a surface on a microscopic scale. The characterization procedure is based on power spectral densities (PSD's) determined from microtopography measurements and subsequent combination of all PSD functions obtained into a single PSD. This provides a surface description over a large bandwidth in the spatial-frequency space, since it allows for the combination of measurements performed at different scales or even with different experimental systems. The surface analysis is accomplished by means of fitting the combined PSD into a model dependent on a reduced set of physically meaningful parameters that quantitatively describe the surface roughness. This enables modeling of PSD's with an arbitrary combination of theoretical models, which can be chosen to match the different roughness contributions that measured PSD's usually contain.

With the results of this analysis procedure it is possible to raise conclusions that would not be available from the plain qualitative observation of the PSD. To illustrate this, the method was applied to coatings with successive layers of $\mathrm{MgF}_{2}$ and $\mathrm{LaF}_{3}$ deposited by ion beam sputtering (IBS) onto fusedsilica (FS) and $\mathrm{CaF}_{2}$ substrates. Employing the proposed analysis procedure provided information on the development of the intrinsic film roughness independently of the substrate characteristics or other roughness contributions such as aggregate formation.

It was also shown that an $A B C$ model is a good approximation for describing the intrinsic roughness of a coating. Also, the aggregates can be adequately modeled with a shifted-Gaussian PSD. The results reveal that the rms roughness and correlation length of the coating increase with increasing number of layers and that the substrate type does not influence this behavior. More specifically, the intrinsic rms roughness increases with the square root of the physical thickness, which is in agreement with other experimental results obtained for fluoride coatings. Concerning the aggregates, the use of the procedure has confirmed that their heights remain approximately constant with increasing number of layers, whereas their sizes increase and their periods increase slightly.

The authors thank Jörg Steinert for his support of the measurements and for fruitful discussions and Aurélien Petit dit Dariel for his contribution to the deposition of fluoride coatings. The authors also gratefully acknowledge the support of the European Commission (TMR network "High-quality UVcoatings," contract ERBFMRX-CT97-0101).

\section{References}

1. U. Stamm, R. Paetzel, I. Bragin, J. Kleinschmidt, D. Basting, and F. Voss, "Recent developments in industrial excimer laser technology," in XI International Symposium on Gas 
Flow and Chemical Lasers and High-Power Laser Conference, D. R. Hall and H. J. Baker, eds., Proc. SPIE 3092, 485-492 (1997).

2. D. Ristau, W. Arens, S. Bosch, A. Duparré, E. Masetti, D. Jacob, G. Kiriakidis, F. Peiro, E. Quesnel, and A. V. Tikhonravov, "UV-optical and microstructural properties of $\mathrm{MgF}_{2}$ coatings deposited by IBS and PVD processes," in Advances in Optical Interference Coatings, C. Amra and H. A. Macleod, eds., Proc. SPIE 3738, 436-445 (1999).

3. J. M. Bennett and L. Mattsson, Introduction to Surface Roughness and Scattering, 2nd ed. (Optical Society of America, Washington, D.C., 1999).

4. J. M. Elson and J. M. Bennett, "Calculation of the power spectral density from surface profile data," Appl. Opt. 34, 201-208 (1995).

5. "Optics and optical instruments-indications in optical drawings," in Draft International Standard ISO 10110 Part 8: Surface Texture, ISO/TC 172/SC 1/WG 2 (International Organisation for Standardisation, Geneva, Switzerland).

6. J. M. Elson and J. M. Bennett, "Relation between the angular dependence of scattering and the statistical properties of optical surfaces," J. Opt. Soc. Am. 69, 31-47 (1979).

7. J. M. Elson, J. P. Rahn, and J. M. Bennett, "Light scattering from multilayer optics: comparison of theory and experiment," Appl. Opt. 19, 669-679 (1980).

8. J. M. Elson, J. P. Rahn, and J. M. Bennett, "Relationship of the total integrated scattering from multilayer-coated optics to angle of incidence, polarization, correlation length, and roughness cross-correlation properties," Appl. Opt. 22, 3207-3219 (1983).

9. J. M. Elson, "Theory of light scattering from a rough surface with an inhomogeneous dielectric permitivity," Phys. Rev. B 30, 5460-5480 (1984).

10. C. Amra and P. Bousquet, "Scattering from surfaces and multilayer coatings: recent advances for a better investigation of experiment," in Surface Measurement and Characterization, J. M. Bennett, ed., Proc. SPIE 1009, 82-97 (1988).

11. C. Amra, J. H. Apfel, and E. Pelletier, "Role of interface correlation in light scattering by a multilayer," Appl. Opt. 31, 3134-3151 (1992).

12. E. L. Church, "Fractal surface finish," Appl. Opt. 27, 15181526 (1988).

13. S. Jakobs, A. Duparré, and H. Truckenbrodt, "Interfacial roughness and related scatter in ultraviolet optical coatings: a systematic experimental approach,” Appl. Opt. 37, 11801193 (1998)

14. E. Quesnel, M. Berger, J. Cigna, D. Duca, C. Pellé, and F. Pierre, "Near-UV to IR optical characterization of $\mathrm{YF}_{3}$ thin films deposited by evaporation and ion beam processes," in Developments in Optical Component Coatings, I. Reid, ed., Proc. SPIE 2776, 366-372 (1996).

15. J. Dijon, E. Quesnel, B. Rolland, P. Garrec, C. Pellé, and J. Hue, "High damage threshold fluoride UV mirrors made by ion beam sputtering," in Laser-Induced Damage in Optical Mate- rials Symposium, Boulder, 1997, G. J. Exharos, A. H. Guenther, M. R. Kozlowski, and M. J. Soileau, eds., Proc. SPIE 3244, 406-416 (1998).

16. J. Y. Robic, Laboratoire d'Electronique de Technologie et d'Instrumentation, Départment Optronique, Grenoble, France (personal communication, 1997).

17. J. C. Stover, Optical Scattering: Measurement and Analysis (McGraw-Hill, New York, 1990).

18. J. M. Bennett and J. H. Dancy, "Stylus profiling instrument for measuring statistical properties of smooth optical surfaces," Appl. Opt. 20, 1785-1802 (1981).

19. A. Duparré and G. Notni, "Multi-type surface and thin film characterization using light scattering, scanning force microscopy and white light interferometry," in Optical Metrology, G. A. Al-Jumailiy, ed., Vol. CR72 of SPIE Critical Review Papers (Society for Photo-Optical Instrumentation Engineers, Bellingham, Wa., 1999), pp. 213-231.

20. A. Duparré, "Light scattering of thin dielectric films," in Thin Films for Optical Coatings, R. E. Hummel and K. H. Günter, eds., Vol. 1 of Handbook of Optical Properties Series (CRC, Boca Raton, Fla., 1995), pp. 273-304.

21. D. Rönnow, "Elastic light scattering by thin films, spectroscopic measurements and analysis," Ph.D. dissertation (Acta Universitatis Upsaliensis, Uppsala, Sweden, 1996).

22. E. L. Church, P. Z. Tackacs, and T. A. Leonard, "The prediction of BRDFs from surface profile measurements," in Scatter from Optical Components, J. C. Stover, ed. Proc. SPIE 1165, 136150 (1989).

23. E. Quesnel, A. Petit Dit Dariel, A. Duparré, J. Ferré-Borrull, and J. Steinert, "DUV light scattering and morphology of ion beam sputtered fluoride coatings," in Advances in Optical Interference Coatings, C. Amra and H. A. Macleod, eds., Proc. SPIE 3738, 410-416 (1999).

24. J. Ferré-Borrull, A. Duparré, and E. Quesnel, "Roughness and light scattering of ion-beam-sputtered fluoride coatings for 193 nm," Appl. Opt. 39, 5854-5864 (2000).

25. G. Rasigni, F. Varnier, M. Rasigni, J. Palmari, and A. Llebaria, "Roughness spectrum and surface plasmons for surfaces of silver, copper, gold, and magnesium deposits," Phys. Rev. B 27, 819-830 (1983).

26. W. H. Press, S. A. Teukolsky, W. T. Vetterling, and B. P. Flannery, Numerical Recipes in $C$, 2nd ed. (Cambridge U. Press, Cambridge, 1992).

27. D. Rönnow, "Interface roughness statistics of thin films from angle-resolved light scattering at three wavelengths," Opt. Eng. 37, 696-704 (1998).

28. S. Bosch, J. Ferré-Borrull, N. Leinfellner, and A. Canillas, "Effective dielectric function of mixtures of three or more materials: a numerical procedure for computations," Surface Sci. 453, 9-17 (2000).

29. A. Duparré, R. Dohle, and H. Müller, "Relation between light scattering and morphology of columnar structured optical thin films," J. Mod. Opt. 37, 1383-1390 (1990). 